\title{
The National Integrative Research Conference, McGill University, March 2019
}

by

$10.17975 /$ sfi-2019-003

Graham Adachi-Kriege, Saashi Bedford, Nadia Blostein, M. Mallar Chakravarty, Gabriel A. Devenyi, Santiago Flores, Megan Kairiss, Sejal Patel, Raihaan Patel, Eric Plitman, Stephanie Tullo, Roy Chen Zhang

\section{The Heritability of Subcortical Structures Using a Twin and Non-Twin Sibling Design}

Nadia Blostein ${ }^{1,7}$, Sejal Patel ${ }^{1,4,5}$, Raihaan Patel ${ }^{1,2}$, Stephanie Tullo ${ }^{1,3}$, Eric Plitman ${ }^{1,6}$, Saashi Bedford ${ }^{1}$, Gabriel A. Devenyi ${ }^{1,6}$, M. Mallar Chakravarty ${ }^{1,2,6}$

${ }^{1}$ Cerebral Imaging Centre, Douglas Mental Health University Institute, Verdun, Canada

${ }^{2}$ Department of Biological and Biomedical Engineering, McGill University, Montreal, Canada

${ }^{3}$ Integrated Program in Neuroscience, McGill University, Montreal, Canada

${ }^{4}$ Campbell Family Mental Health Research Institute, Centre for Addiction and Mental Health, Toronto, Canada

${ }^{5}$ Institute of Medical Science, University of Toronto, Toronto, Canada

${ }^{6}$ Department of Psychiatry, McGill University, Montreal, Canada

${ }^{7}$ Faculty of Arts \& Science, McGill University, Montreal, Canada

The increase in open access and large-scale neuroimaging datasets, such as the UK BioBank, has accelerated the use of structural neuroimaging to better understand how genetic variation may impact brain structure 1 . However, in many imaginggenetics studies, the heritability of the quantitative phenotype under study is often a prerequisite for its use in future analyses (such as genome-wide association studies). The current work extends previous work from our group examining the heritability of the hippocampus and its subfields to examine the heritability of subcortical structures, specifically, the thalamus, globus pallidus, and striatum. We use structural magnetic resonance imaging (MRI; 3T; T1w; $0.7 \mathrm{~mm}$ voxel size) data from healthy adult twins (monozygotic and dizygotic) and non-twin siblings who participated in the Human Connectome Project (HCP; $n=1086$ participants) 2. Images were preprocessed using the minc-bpipelibrary (https://github.com/CobraLab/minc-bpipe-library/blob/ master/minc-library.bpipe), and subcortical structures were labeled using the Multiple Automatically Generated Templates
(MAGeT) brain segmentation algorithm3 and the CoBrA Lab subcortical atlases 4 (https://github.com/CobraLab/atlases/ tree/master/5-atlas-subcortical) to obtain volume estimates. Output subcortical segmentations from MAGeT brain were manually quality controlled for accuracy, with 1008, 1007 and 1009 subjects passing for the globus pallidus, the thalamus and the striatum, respectively. The OpenMx package (version 2.12.2) in $\mathrm{R}$ (version 3.5.1) was used to compute heritability estimates of subcortical volumes through structural equation modeling 5. A univariate model was used to compute the heritability of subcortical structure volume across all subjects. Results showed significant genetic effects $(p<0.001)$ and yielded heritability estimates ranging from $74 \%$ (for both left and right globus pallidus) to $88 \%$ (right striatum). Estimates were consistent across hemispheres for the globus pallidus (74\% for both the left and right hemispheres) and the thalamus ( $88 \%$ for both the left and right hemispheres). The Pearson correlation between total brain volume and subcortical volume ranged from 0.71 (right globus pallidus) to 0.84 (right thalamus). A bivariate model was used to compute the shared heritability between total brain volume and subcortical volume. Results showed significant genetic effects ( $p<0.001$ ) and yielded heritability estimates ranging from $75 \%$ (left and right striatum) to $88 \%$ (left globus pallidus). The genetic correlation between total brain volume and subcortical volume ranged from 0.76 (right globus pallidus) to 0.86 (right thalamus). These results suggest that the phenotypic correlation between subcortical volumes is largely due to genetic effects. Such findings can lead to a better understanding of the significance of genetic risk factors in subcortical diseases. 


\section{The Orangutan Crisis: The Role of Poaching and the Illegal Primate Trade in the Bornean Orangutan Population Decline}

Santiago Flores

The Bornean orangutan (Pongo pygameus) is currently listed by the IUCN as a critically endangered species and their populations will continue to decrease unless effective conservation measures are enacted quickly. The objective of this paper is to understand the underlying causes behind the Bornean orangutan population decline specifically focusing on the role of poaching, as well as to discuss current and alternative conservation strategies needed for the protection of this endangered species. The findings of this article were derived from a literature review encompassing over 30 peer-reviewed articles related to orangutan conservation and the illegal wildlife trade. Bornean orangutan populations are mainly threatened by massive destruction of their habitat and poaching. Every year, hundreds of infant orangutans are taken from their habitat and put into the illegal wildlife trade to be sold as pets or entertainment for zoos. High levels of corruption and weak law enforcement from Indonesian authorities are the major factors enabling the trade of this species. Conservation strategies have focused on (I) the confiscation, rehabilitation, and reintroduction of orangutans into the wild and (2) the protection of orangutan habitat from deforestation and hunting. Successful conservation will primarily aim to implement strong and effective law enforcement including the prosecution of criminals, prioritize funding for habitat management and protection over rehabilitation programs, address the needs of local people, and develop orangutan-friendly management programs for nonprotected forest areas.

\section{Topic Modelling: Uncovering Hidden Meaning in Text Data Megan Kairiss, Graham Adachi-Kriege}

Say you'd like to examine the biological and political factors relating to the potential extinction of an animal. Where would you begin? You might start by using your search engine to find articles on the species. But this would likely find articles related to the keyword that you searched, rather than the target of the research question. What if you want to find articles that relate to your broad research goals, and able to search for documents that might have a mixture of research on both the animal and politics? This frames the value of an algorithm that allows you to uncover the hidden structure of text corpora, Topic Modelling. Topic Modelling is an unsupervised machine learning algorithm that allows us to examine large amounts of documents without the expense of human labor. The hidden latent structure of documents can be explained by topic distributions, where each topic is defined as a distribution over the vocabulary of the documents. This makes sense to us as humans, as we describe topics as representing the themes and ideas of texts. A human reading a news article about the stock market might identify the main themes as "corporate", "market", and "value", but would be less likely to describe the article with a topic like "surfing". The "topics" in topic modelling are exactly the same, operating under the premise that words belonging to the same theme are likely to co-occur across documents.

\section{Nelson, Siegel...Diophantus? A Novel Approach to Term Structure Modeling}

Roy Chen Zhang

We present a more flexible alternative to the functional approximations of principal component analysis in estimating term structures first proposed by Nelson and Siegel (1987) and extended by Svensson (1994). We argue that the added complexity brought about by a second horizontal scaling factor $\lambda 2$ in the Nelson-SiegelSvensson model is unnecessary, and instead use Diophantine approximation techniques to derive a more flexible alternative that allows fitted curves to be humped without the need for a second horizontal scaling factor. We then show that the proposed Diophantine model can be readily extended to allow for even more variation in term structures, and demonstrate a conversion method from Diophantine factor weightings to the more intuitive Nelson-Siegel factor weightings. The proposed model is evaluated against current alternatives, comparing performance through accuracy, computational intensity, and robustness. Finally, we conclude by briefly discussing the tractability of the Diophantine family of term structure models in stochastic forecasting. 